\title{
Genotypic variation in disease susceptibility among cultured stocks of elkhorn and staghorn corals
}

\author{
Margaret W Miller Corresp., ${ }^{1,2}$ ， Philip J Colburn ${ }^{3}$, Emma Pontes ${ }^{3}$, Dana E Williams ${ }^{1,3}$, Allan J Bright ${ }^{1,3}$, \\ Xaymara Serrano ${ }^{3,4}$, Esther C Peters ${ }^{5}$ \\ ${ }^{1}$ Southeast Fisheries Science Center, NOAA-National Marine Fisheries Service, Miami, Florida, United States \\ 2 SECORE International, Miami, Florida, United States \\ 3 Rosenstiel School of Marine and Atmospheric Science, University of Miami, Miami, Florida, United States \\ 4 Atlantic Oceanographic and Meterological Laboratory, NOAA Oceanic and Atmospheric Research, Miami, FL, USA \\ 5 Department of Environmental Science and Policy, George Mason University, Fairfax, VA, USA \\ Corresponding Author: Margaret W Miller \\ Email address: m.miller@secore.org
}

Disease mortality has been a primary driver of population declines and the threatened status of the foundational Caribbean corals, Acropora palmata and A. cervicornis. There remain few tools to effectively manage coral disease. Substantial investment is flowing into in situ culture and population enhancement efforts, while disease takes a variable but sometimes high toll in restored populations. If genetic resistance to disease can be identified in these corals, it may be leveraged to improve resistance in restored populations and possibly lead to effective diagnostic tests and disease treatments. Using a standardized field protocol based on replicated direct-graft challenge assays, we quantified this important trait in cultured stocks from three field nurseries in the Florida Keys. Field tests of 12 genotypes of $A$. palmata and 31 genotypes of $A$. cervicornis revealed significant genotypic variation in disease susceptibility of both species measured both as risk of transmission (percent of exposed fragments that displayed tissue loss) and as the rate of tissue loss $\left(\mathrm{cm}^{2} \mathrm{~d}^{-1}\right)$ in fragments with elicited lesions. These assay results provide a measure of relative disease resistance that can be incorporated, along with consideration of other important traits such as growth and reproductive success, into restoration strategies to yield more resilient populations. 
1

2

3

4 Genotypic variation in disease susceptibility among cultured stocks of elkhorn and staghorn

5 corals

6

7 Margaret W. Miller ${ }^{1,2}$, Philip J. Colburn ${ }^{3}$, Emma Pontes $^{3}$, Dana E. Williams ${ }^{1,3}$, Allan J. Bright ${ }^{1,3}$,

8 Xaymara Serrano ${ }^{3,4}$, Esther C. Peters ${ }^{5}$

9

10

11

12
1) Southeast Fisheries Science Center, NOAA-National Marine Fisheries Service, Miami FL, USA;

2) Corresponding Author, current contact: SECORE International, Miami FL, USA, m.miller@secore.org

3) Rosenstiel School of Marine and Atmospheric Sciences, University of Miami, Miami FL, USA

4) Atlantic Oceanographic and Meteorological Laboratory, NOAA-Oceanic and Atmospheric Research, Miami FL USA

5) Department of Environmental Science and Policy, George Mason University, Fairfax, VA, USA 
Abstract:

21 Disease mortality has been a primary driver of population declines and the threatened status of

22 the foundational Caribbean corals, Acropora palmata and A. cervicornis. There remain few tools

23 to effectively manage coral disease. Substantial investment is flowing into in situ culture and

24 population enhancement efforts, while disease takes a variable but sometimes high toll in

25 restored populations. If genetic resistance to disease can be identified in these corals, it may be

26 leveraged to improve resistance in restored populations and possibly lead to effective diagnostic

27 tests and disease treatments. Using a standardized field protocol based on replicated direct-graft

28 challenge assays, we quantified this important trait in cultured stocks from three field nurseries

29 in the Florida Keys. Field tests of 12 genotypes of $A$. palmata and 31 genotypes of $A$.

30 cervicornis revealed significant genotypic variation in disease susceptibility of both species

31 measured both as risk of transmission (percent of exposed fragments that displayed tissue loss)

32 and as the rate of tissue loss $\left(\mathrm{cm}^{2} \mathrm{~d}^{-1}\right)$ in fragments with elicited lesions. These assay results

33 provide a measure of relative disease resistance that can be incorporated, along with

34 consideration of other important traits such as growth and reproductive success, into restoration

35 strategies to yield more resilient populations. 
41

42

43

44

45

46

47

Introduction:

Disease constitutes an existential threat to coral persistence (e.g., Walton, Hayes et al. 2018), and this threat is exacerbated by its worsening with thermal stress (Randall and van Woesik 2015). Studies correlating various life history traits with disease susceptibility have shown that the family Acroporidae, with its high skeletal growth rates and low investment in immunity, is particularly susceptible to disease (Palmer, Bythell et al. 2010, Díaz and Madin 2011). The two species of Acropora native to the Caribbean, A. palmata and A. cervicornis, are both designated as critically endangered by the IUCN and threatened under the US Endangered Species Act (ESA) with disease cited as a primary driver of their high extinction risk (Aronson and Precht 2001, NMFS 2006). The strong association of climate change related thermal stress and disease is also well-documented in both these species (Muller, Rogers et al. 2008, Randall and van Woesik 2015), lending little expectation of abatement under expected continued warming.

Legal mandates imposed by the ESA listing as well as recognition of persistent patterns of population decline have yielded increasing investment and success in population enhancement for A. cervircornis and, more recently, A. palmata (Lirman and Schopmeyer 2016, Miller, Kerr et al. 2016). Currently, population enhancement efforts focus on fragment-based (clonal) propagation methods, yielding relatively low genotypic diversity (e.g., up to 100 genotypes utilized in a regional population enhancement program) and potentially higher disease susceptibility (Altizer, Harvell et al. 2003). However, wild remnant populations for these species often display even lower genotypic diversity (e.g., individual reefs occupied by a single genotype; Baums, Miller et al. 2006). Disease takes a variable but sometimes high toll on restored populations, at a level comparable to that on wild remnant populations (Miller, Lohr et 
64 al. 2014). The current lack of tools for management or mitigation of disease impacts in either

65

66

67

68

69

70

71

72

73

74

75

76

77

78

79

80

81

82

83

84

wild or restored populations underscores the potential benefits to identifying natural disease resistance within populations. Indeed, Vollmer and Klein (2008) identified $6 \%$ of $A$. cervicornis genotypes as being resistant to disease in a wild population in Panama based on field surveys and field-based challenge assays and, more recently, Muller et al. (2018) identified 27\% of $A$. cervicornis genotypes in nursery culture in the lower Florida Keys as disease resistant in laboratory challenge assays using a homogenized inoculant. No published studies have tested such resistance in A. palmata.

Identifying genotypes with specific disease susceptibility or resistance traits could provide an important tool in the quest to build more resilient, recovered populations of these threatened species. Also, the quantification of these disease susceptibility traits among genotypes is a pre-requisite to further investigation of underlying biological and/or genomic mechanisms (e.g., Libro and Vollmer 2016) that may eventually lead to effective diagnostic tests and disease treatments. Thus, we performed field challenge assays to quantify disease susceptibility or resistance in a range of genotypes from stocks cultured in three upper Florida Keys coral nurseries.

Methods:

Susceptibility assays:

We performed field challenge assays according to a protocol that was previously illustrated and published to facilitate standard trait quantification by other researchers or nursery operators (Miller and Williams 2016). All assays were performed on segregated, experimental 
86

87

88

coral nursery 'trees' (Nedimyer, Gaines et al. 2011) at the nursery operated by the Coral

Restoration Foundation off Tavernier, Florida, USA. All nursery stocks had been previously genotyped (via microsatellites or direct sequencing of mitochondrial or nuclear genes; see Tables S1 and S2) and tracked through propagation via best practices (Johnson, Lustic et al. 2011). Briefly, replicate fragments of the tested genotypes were collected from the nursery population and deployed to the experimental 'trees' in a segregated area of the nursery. After at least two weeks' stabilization period, actively diseased fragments were collected from the nursery population ('inoculants'; all A.cervicornis) and attached securely to the apparently healthy test fragment with cable ties. The genotype of inoculant fragments was not recorded or controlled, though multiple genotypes of inoculant were used in each trial. However, we applied all inoculants haphazardly across replicates of different genotypes to minimize potential bias; within each trial, the sequential collection of inoculant fragments (which was clumped by the nursery tree of origin and, hence, genotype) was distributed across replicate test fragments of different genotypes.

Hereafter, we use the term 'transmission' to refer to the appearance of tissue loss signs on a test fragment after the application of an inoculant, though the use of this term does not imply anything about the specific mechanism or pathogen that might have caused the tissue loss. Bacteria, ciliates, and suspect viruses have all been detected in cases of Caribbean Acropora tissue-loss disease (Miller, Lohr et al. 2014, Sweet, Croquer et al. 2014, Peters, Pers. Obs.). However, the responsible biotic pathogen may shift over time (Sutherland, Berry et al. 2016) and/or a dysbiosis (i.e., alteration of normal microbiome) or a noninfectious abiotic factor (e.g., elevated or lowered sea temperatures or chemical contaminant) (Lesser, Bythell et al. 2007) may be the dominant agents of disease in A.palmata and A.cervicornis. 

test fragments, photographed, and elicited lesions measured) on day 1, 3, and 5 after

111 implementation (day 0) and the trial was completed on day 7, when inoculants were removed and

112 discarded, along with all lesioned fragments. Slight deviation in timing of surveys was dictated 113 by extraneous events (weather or other disturbance). In accordance with permit conditions, test

114 fragments that did not show signs of tissue loss were either discarded or remained quarantined 115 for several more weeks and then transferred back to nursery stocks or laboratory studies.

116 Sixteen $A$. cervicornis and six A. palmata genotypes were screened in two 1-week trials

117 between July-August 2016, with an additional two trials run in July-August 2017 (additional 14

118 A. cervicornis and six A.palmata genotypes). Three response parameters were analyzed and

119 compared among genotypes in the transmission assays for each year and species separately.

120 Risk of transmission $\left(7 \mathrm{~d}^{-1}\right)$ was expressed as the proportion of assay replicates in which tissue-

121 loss lesions were observed ( $n=7-10$ for genotypes tested in 2016, $n=10$ for all 2017 genotypes).

122 This proportion for each genotype was compared, via Pearson Chi-Squared tests, to an

123 'expected' value based on the pooled population of all the genotypes assayed in the same year.

124 Second, the time to first appearance of tissue loss (i.e. survey day on which lesion was first observed) was averaged for transmitted replicates of each genotype with at least three lesioned replicates and compared via Kruskall-Wallis ANOVA. Third, the length of all elicited lesions

127 was measured with a ruler in situ at each survey. The ending length of each lesion (i.e., at day 7)

128 was divided by the number of days since the lesion was first observed. These rates (i.e., 129 progression of tissue-loss margin; $\mathrm{cm} \mathrm{d}^{-1}$ ) were averaged for each genotype with at least three 130 lesioned replicates and compared among genotypes via a Kruskal-Wallis ANOVA. To obtain 131 higher replication for the latter two parameters in 2016 (when overall transmission rates were 
132 lower than 2017), fragments that did not experience tissue loss in the initial trial were re-exposed

133 to a second inoculant trial. Replicates that showed elicited lesions in this repeat assay were

134 included in the reported tissue-loss rate and time to transmission data but were not included in

135 the risk of transmission score (since they had experienced added inoculation 'dose').

136

137 Inoculant characterization and controls:

138 A. cervicornis fragments were used as inoculants in the assays for both species because

139 no disease was observed in the background nursery A.palmata population. Since there are no

140 definitive field diagnostic tools for $A$. cervicornis disease identification, we performed basic

141 histopathological observations to characterize the disease inoculants used in our assays. On day 0

142 of each transmission trial, additional diseased fragments (comparable to those used as inoculants;

$143 \mathrm{n}=13$ A. cervicornis in 2016; $\mathrm{n}=3$ in 2017) were fixed in a solution of one part zinc-buffered

144 formalin (Z-Fix, Anatech, Ltd) diluted with four parts seawater. Samples were shipped to the

145 Histology Laboratory at George Mason University for processing and slide reading according to

146 the protocols described in Miller et al. (2014). Additional histoslides cut from one representative

147 sample from each year were treated with the eubacterial probes EUB338-I and EUB338-II,

148 tagged with AlexaFluor 488 and CY3 fluorescent dyes (Creative Bioarray, New York),

149 respectively, and a nonsense probe (nonEUB338) to investigate suspect bacteria. After applying

150 the probes to separate deparaffinized and hydrated slides in a $45 \%$ formamide hybridization

151 buffer solution, the samples were heated to $55^{\circ} \mathrm{C}$ for 2 hours, rinsed with wash buffer, and

152 coverslipped with Vectashield hardset mounting medium with DAPI (Vector Laboratories, Inc.,

153 California) before examining with a Leica DM2000 fluorescence microscope and integrated

154 camera. 

cervicornis, with unknown effect on its potential infectivity. We chose the visually most active

157 tissue-loss margins we could find (i.e., widest margins of bright white exposed skeleton and 158 ragged or 'sloughing' appearance of the tissue margin) as inoculants. We applied all inoculants 159 haphazardly across replicates of different genotypes to minimize potential bias from 160 differentially infective inoculants. In 2017 we also used photographs and measured lesion size at 161 the beginning and end of each trial to verify the progression of tissue loss on the inoculant 162 fragments.

Note that the purpose of this study was to quantify tissue-loss disease susceptibility as a phenotypic trait of specific genotypes, not to characterize the potential pathogen nor transmission mechanism per se. Hence, a full slate of 'healthy inoculant' control treatments was not undertaken. Nonetheless, a small number of 'control' assays was conducted in 2016 using

167 healthy-looking A. cervicornis 'inoculants' paired with one fragment each of four test genotypes of each species. These $A$. palmata controls $(\mathrm{n}=4)$ represent healthy allografts (i.e., healthylooking A. cervicornis 'inoculants' applied to an A. palmata test fragment) to detect potential allo-rejection response independent of disease exposure. Marine Sanctuary permit \# FKNMS-2016-024-A1.

Results:

None of the 'healthy control' assays conducted in 2016, including the allograft controls, resulted in development of tissue-loss lesions or any visually observable anomalies on the test

177 fragments, though minor abrasion on protruding calices was sometimes observed (Fig S1 A-B). 
For the 2017 trials, we noted progression of the tissue-loss margin of each inoculant and

$17981 \%$ of them lost noticeable tissue during the 7-day trial ('active' inoculants). If the other 19\%

of replicates involving inoculants without observable tissue loss ('inactive' inoculants) are

181

excluded, the range of transmission risk among tested genotypes was 38-100\%, compared to $30-$

$100 \%$ when all replicates were included. Also, we observed multiple instances (5 out of 27 ) in

which an inactive inoculant was associated with transmission, suggesting that lack of progressing

tissue loss did not preclude its ability to cause tissue loss (i.e., serving as an effective inoculant).

For these reasons, we included all replicates in results reported here. We observed greater

virulence of the inoculant condition, as manifested by a higher overall risk of transmission, in the

2017 trials (56\% transmission for A. cervicornis, 82\% transmission for A.palmata) than in 2016

188

189

190

191

192

193

194

195

196

197

198

199

200

(30\% for each species).

As expected, considerable variability was observed in the characterization of the inoculant samples submitted for histological examination. Eleven of these 13 inoculant samples from 2016 were characterized as white band disease (WBD) from their gross signs (i.e., the disappearance of coral tissue along a smooth margin, mostly starting from the base of the branch, although two of these had tip lesions that may have been caused by predation). These fragments had diverse microorganisms trapped in the protective agarose layer that was applied over the tissue-loss margins prior to decalcification and processing, including Symbiodinium-containing ciliates adjacent to sites wheregastrodermal cells had been stripped from the mesoglea, then the epidermal cells lysed or sloughed off (Fig S2A). However, other areas lacked ciliates. Intracellular Rickettsiales-like organisms (RLOs) in mucocytes of polyp structures (Fig S2B), but only one of these (grossly) WBD samples had excess mucus production in epidermal mucocytes. Tissues also varied in the formation of single-cell necrosis or apoptosis and degraded 
201 cell spherules at the tissue-loss margins. The remaining two samples from 2016 and all three

202 sampled from 2017 were grossly characterized as rapid tissue loss, (RTL), having acute patchy

203 sloughing of tissues off the branches; denuded skeleton was bright white, lacking fouling

204 organisms. These RTL samples were in poorer condition, with more epidermal mucus production

205 (Fig S2C) and suspect bacteria (based on Giemsa staining) in the mucus (Fig S2D), some

206 ciliates, and lysing and necrosis of atrophied epithelia. Along the tissue-loss margin, portions of

207 the calicodermis consisted of thin columnar, hypertrophied cells with abundant eosinophilic

208 apical granules of coral acid-rich proteins (CARPs) (Fig. S2E). The tissues displayed larger foci

209 of single-cell necrosis or apoptosis in basal and surface body walls (Fig S2F) and formed

210 necrotic cell spherules at the tissue-loss margin (Fig S2G). Fewer ciliates were associated with

211 the 2017 samples. However, as has been found in Caribbean acroporid samples affected by either

212 type of tissue-loss pattern (Peters, pers. obs.), sections through tentacles revealed abundant

213 zooxanthellae in the gastrodermis (as expected), but the gastrodermal cells were also variably

214 filled with minute turquoise-staining (with Giemsa) specks, in some places being released

215 through the apical surfaces of the gastrodermal cells into the gastrovascular cavity fluid. The

216 gastrodermis areas in the tentacles with the densest aggregations of these specks had fewer algal

217 cells (Fig S2H).

218 The FISH procedure showed positive staining with the bacterial probes by abundant

219 minute coccoid or other-shaped cells on the surfaces of necrotic or apoptotic areas (Fig S3A-D).

220 RLOs were present in the mucocytes of the tentacles, oral disc, actinopharynx epidermis, and

221 cnidoglandular bands, with similar densities of infected cells in 2017 samples as found in all of

222 the 2016 samples. The FISH probes revealed differences in the distribution of bacteria between

223 the samples, however, as well as morphologies of the bacteria (Fig S3A-D). The most degraded 
224 tissues along the tissue-loss margins had abundant minute bacteria that were labeled by both the 225 EUB338-I and EUB338-II probes (Fig S3E-F).

Despite variable inoculant virulence, significant variation was observed among genotypes

227 of each species in risk of transmission (Fig 1A-B). In 2016, four of 16 A. cervicornis genotypes

228

229

230

231

232

233

234

235

236

237

238

239

240

241

242

243

244

245

246

and one of six A. palmata genotypes had significantly higher rates of transmission than expected (Pearson Chi-squared test, $\mathrm{p}<0.05$; Fig 1A). Two additional A. cervicornis genotypes and one additional $A$. palmata genotype appeared relatively resistant to disease (i.e., zero tissue loss in $\mathrm{n}=7-10$ replicate exposures) in 2016, although the power of the Pearson Chi Squared tests was not adequate to distinguish these from the pooled population transmission risk of $30 \%$ with $95 \%$ confidence (Fig 1A). In 2017, three of 14 tested A. cervicornis genotypes had significantly higher risk of transmission than the population expectation of $56 \%$, whereas none of the six tested A. palmata genotypes differed significantly from the $82 \%$ expected transmission risk (Fig 1B).

Mean tissue-loss rates for elicited $A$. cervicornis lesions ranged from $\sim 0.5$ to $4 \mathrm{~cm} \mathrm{~d}^{-1}$ in 2016 and from $\sim 1$ to $7.6 \mathrm{~cm} \mathrm{~d}^{-1}$ in 2017-tested genotypes (Fig 1C-D). This represented statistically significant variation in 2016 ( $p=0.022$, Kruskal Wallis ANOVA) but not in 2017 $(\mathrm{p}=0.135)$. Tissue-loss rates in A. palmata did not show significant variation among genotypes in either year (Fig 1C-D). Similarly, 'days to transmission' did not differ significantly among genotypes for either species tested in either 2016 or 2017 (Fig 1 E-F).

Discussion:

The prior work on which this study was based had shown $6 \%$ of 49 wild A. cervicornis genotypes in Panama to be 'resistant' to what the authors referred to as White Band Disease (Vollmer and Kline, 2008). This designation of resistance was based on $\sim$ quarterly surveillance 
247 of disease prevalence in this wild population over a 1-year period and a score of zero

248 transmission during a 3-5-day challenge assay with replication of five. A more recent study

249 (Muller, Bartels et al. 2018) using laboratory challenge to a homogenized tissue inoculant (and

250 replication of 5 to 7 ) determined $27 \%$ of $A$. cervicornis genotypes (four of 15 ) from a nearby

251 coral nursery (lower Florida Keys) were resistant under background conditions, although most

252 lost resistance during acute heat stress/bleaching. Only one of these four genotypes remained

253 disease-resistant when bleached by a warm thermal anomaly affecting this nursery in Sept 2015

254 (Muller, Bartels et al. 2018).

255

Our results in 2016 suggested that $12.5 \%$ of the assayed $A$. cervicornis and $17 \%$ of $A$.

256

257

258

259

260

261

262

263

264

265

266

267

268

palmata genotypes were resistant (i.e., zero transmission of disease signs in $n=8$ or 9 replicate

exposures). However, none of the screened genotypes showed zero transmission in 2017. This

shift to less observed resistance in the second year is unlikely to be due to differential thermal

stress/bleaching as no substantial difference in thermal stress (i.e., overall average at nearby

Molasses Reef between 1 July-20 Aug was 29.8 for 2016, 29.9 for 2017;

https://www.ndbc.noaa.gov/station_history.php?station=mlrf1) and no bleaching was observed

in the nursery populations during either year. Instead, we suggest that this pattern resulted from either including more susceptible test genotypes in 2017 and/or the presence of more virulent disease condition in the background population and hence the inoculants used in 2017.

Something in those inoculants caused thick mucus discharge (whether bacterial or toxin mediated) and was acutely degrading the tissues to rapidly form large areas of single-cell necrotic or apoptotic changes. The differences revealed by FISH suggest that the microbial communities were potentially different, not only in the quantity of bacteria present, but also in 
269 species present, as revealed by their morphologies. Molecular methods are needed to understand

270 these changes, but may be difficult and expensive to apply during assays.

This emphasizes the likelihood that field-collected inoculants, though visually similar and collected from the same location/population, may not represent the same condition. Our assays

273 relied on visual determination of active disease signs to identify effective inoculants (due to the

274 lack of available reliable field diagnostic tools for Acropora diseases) and we did not control nor

275

276

277

278

279

280

281

282

283

284

285

286

287

288

289

290 track the genotypes of the inoculants. Nonetheless, the lack of allogenic response in the healthy controls and the haphazard pairing of inoculants across test genotypes with relatively high replication make it quite unlikely that the statistically significant variability in disease susceptibility observed within each assay trial was spuriously caused by the uncontrolled genotype of the inoculant. It does, however, suggest that results of this protocol should be interpreted as indicating relative disease susceptibility, rather than absolute resistance, and confidence in this relative susceptibility ranking is highly dependent on replication. Additional histological and molecular microbiological studies of coral fragments used in repeat susceptibility assays under different conditions would also improve the interpretation of 'relative resistance.'

Contrasting tissue-loss rates have been cited in past literature as potentially diagnostic signs for particular coral diseases (e.g., Richardson, Goldberg et al. 1998). However, the current results indicate that tissue-loss rate can be characteristic to particular genotypes within the same disease event. Interestingly, a few A. cervicornis genotypes showed tissue-loss rate contrasting with the risk of disease signs transmission, suggesting the possibility of multiple mechanisms of resilience to disease effects. For example, genotypes C3, C17, and C22 showed higher than 
291 expected risk of transmission, but relatively low tissue-loss rates, perhaps indicative of a strategy

292 of disease (tissue loss) tolerance rather than resistance.

293 Disease resistance, along with other beneficial traits, is commonly discussed as a tool to

294 leverage greater resilience and prospects of long-term recovery in restored coral populations.

295 The characterization of a range of fitness traits (growth, reproduction, as well as disease or

296 thermal resistance) is important to consider in selecting genotypes for propagation as ecological

297 tradeoffs or unanticipated environmental stressors may yet compromise the success of disease-

298 resistant individuals. Field studies include cases where disease resistance is associated with both

299 positive (e.g., high growth rates) and negative (reduced thermal tolerance) traits (Shore-Maggio,

300 Callahan et al. 2018). It may be appropriate to preferentially include a disease-resistant genotype

301 in an outplanting design (e.g., inclusion across all sites), but it must be incorporated in diverse

302 patches. Diverse provenance of outplanted coral populations gives the greatest likelihood of

303 successful fertilization and larval production as well as greatest adaptability of these offspring.

304 This quantification of variation in disease-resistance can also serve as a basis for mechanistic

305 studies, potentially leading to much-needed effective disease management tools.

307 Conclusion:

This study has documented genotypic variation in disease susceptibility, as evidenced by risk of transmission and tissue loss rates, in both Caribbean Acroporid species that are highly

310 targeted in population enhancement efforts. This provides a context for continuing genomic

311 investigations of mechanism and potential development of diagnostics (Traylor-Knowles and

312 Young, in progress). In combination with quantification of other phenotypic traits, this result also 
313 provides the potential to leverage this trait in restored populations to increase resilience, but in a

314 strategy that maintains genetic diversity.

316 Acknowledgements: The collaboration and support of the three regional coral nurseries operated

317 by the Coral Restoration Foundation, the Florida Fish and Wildlife Research Institute, and the

318 University of Miami are gratefully acknowledged. Field assistance from R. Pausch, and A.

319 Peterson is also greatly appreciated. K.A. Cobleigh, E.V. Mazur, and V.T. Nguyen prepared the 320 tissue samples for histopathological examination.

References Cited:

323

324

325

326

327

328

329

330

331

332

333

Altizer, S., D. Harvell and E. Friedle (2003). "Rapid evolutionary dynamics and disease threats to biodiversity." Trends in Ecology \& Evolution 18(11): 589-596.

Aronson, R. B. and W. F. Precht (2001). "White-band disease and the changing face of Caribbean coral reefs." Hydrobiologia 460(1-3): 25-38.

Baums, I. B., M. W. Miller and M. E. Hellberg (2006). "Geographic variation in clonal structure in a reefbuilding Caribbean coral, Acropora palmata." Ecological Monographs 76(4): 503-519.

Díaz, M. and J. Madin (2011). "Macroecological relationships between coral species' traits and disease potential." Coral Reefs 30(1): 73-84.

Johnson, M. E., C. Lustic, E. Bartels, I. B. Baums, D. S. Gilliam, L. Larson, D. Lirman, M. W. Miller, K. Nedimyer and S. Schopmeyer (2011). Caribbean Acropora Restoration Guide: Best Practices for Propagation and Population Enhancement. Arlington, VA., The Nature Conservancy: 54. 
334 Lesser, M. P., J. C. Bythell, R. D. Gates, R. W. Johnstone and O. Hoegh-Guldberg (2007). "Are infectious diseases really killing corals? Alternative interpretations of the experimental and ecological data." Journal of Experimental Marine Biology and Ecology 346(1-2): 36-44.

Libro, S. and S. V. Vollmer (2016). "Genetic Signature of Resistance to White Band Disease in the Caribbean Staghorn Coral Acropora cervicornis." PLoS ONE 11(1): e0146636.

Lirman, D. and S. Schopmeyer (2016). "Ecological solutions to reef degradation: optimizing coral reef restoration in the Caribbean and Western Atlantic." PeerJ 4: e2597.

Miller, M. W., K. Kerr and D. E. Williams (2016). "Reef-scale trends in Florida Acropora spp. abundance and the effects of population enhancement." PeerJ 4: e2523.

Miller, M. W., K. E. Lohr, C. M. Cameron, D. E. Williams and E. C. Peters (2014). "Disease dynamics and potential mitigation among restored and wild staghorn coral, Acropora cervicornis." PeerJ 2: e541.

Miller, M. W. and D. E. Williams (2016). "A standard field protocol for testing relative disease resistance in Acropora palmata and Acropora cervicornis." PeerJ Preprints 4: e2668v2661.

Muller, E. M., E. Bartels and I. B. Baums (2018). "Bleaching causes loss of disease resistance within the threatened coral species Acropora cervicornis." eLife 7: e35066.

Muller, E. M., C. S. Rogers, A. S. Spitzack and R. van Woesik (2008). "Bleaching increases likelihood of disease on Acropora palmata (Lamarck) in Hawksnest Bay, St John, US Virgin Islands." Coral Reefs 27: 191-195.

Nedimyer, K., K. Gaines and S. Roach (2011). "Coral tree nursery: an innovative approach to growing corals in an ocean-based field nursery." AACL Bioflux 4(4): 442-446.

NMFS (2006). "Endangered and Threatened Species:Final Listing Determinations for Elkhorn Coral and Staghorn Coral." Federal Register 71: 26852-26861.

Palmer, C. V., J. C. Bythell and B. L. Willis (2010). "Levels of immunity parameters underpin bleaching and disease susceptibility of reef corals." The FASEB Journal 24(6): 1935-1946. 
359 Randall, C. J. and R. van Woesik (2015). "Contemporary white-band disease in Caribbean corals driven

360

361

362

363

364

365

366

367

368

369

370

371

372

373

374

375

376

377 by climate change." Nature Clim. Change 5(4): 375-379.

Richardson, L. L., W. M. Goldberg, R. G. Carlton and J. C. Halas (1998). "Coral disease outbreak in the Florida Keys: plague type II." Rev. Biol. Trop 46(Supl 5): 187-198.

Shore-Maggio, A., S. M. Callahan and G. S. Aeby (2018). "Trade-offs in disease and bleaching susceptibility among two color morphs of the Hawaiian reef coral, Montipora capitata." Coral Reefs 37(2): 507-517.

Sutherland, K. P., B. Berry, A. Park, D. W. Kemp, K. M. Kemp, E. K. Lipp and J. W. Porter (2016). "Shifting white pox aetiologies affecting Acropora palmata in the Florida Keys, 1994-2014." Philosophical Transactions of the Royal Society of London B: Biological Sciences 371(1689).

Sweet, M. J., A. Croquer and J. C. Bythell (2014). "Experimental antibiotic treatment identifies potential pathogens of white band disease in the endangered Caribbean coral Acropora cervicornis." Proc. R. Soc. B 281(1788): 20140094.

Vollmer, S. V. and D. I. Kline (2008). "Natural disease resistance in threatened staghorn corals." PLoS ONE 3: e3718. doi:3710.1371/journal.pone.0003718.

Walton, C. J., N. K. Hayes and D. S. Gilliam (2018). "Impacts of a Regional, Multi-Year, Multi-Species Coral Disease Outbreak in Southeast Florida." Frontiers in Marine Science 5(323). 


\section{LEGENDS:}

379 Figure 1: Results of disease susceptibility assays conducted in 2016 (A, C, E) and 2017 (B, D, F)

380 showing A-B) the risk of transmission (i.e. the proportion of replicate ramets of each genotype in

381 which the elicitation of a tissue loss lesion was observed following the application of an

382 inoculant fragment); C-D) the average rate of progression of tissue loss for genotypes with at

383 least three responding replicates ( $p$-values in C show significance of Kruskall-Wallace ANOVA

384 for each species; variation in D is not significant for either species); and E-F) the average time

385 until a lesion was observed for genotypes with at least three responding replicates. Genotype

386 designations (given along the $\mathrm{X}$-axis) are identified in Table S1. The number of replicates for

387 each genotype is given inside or above each bar while error bars indicate $+1 \mathrm{SE}$. In A and B,

388 dashed lines show the pooled population risk of infection for each species, asterisks indicate

389 genotypes that differed significantly from this expected transmission risk, and crosses indicate

390 zero transmission observed for that replicate (though power for the Pearson Chi-Squared test was

391 inadequate to 


\section{Figure 1 (on next page)}

Results of disease susceptibility assays conducted in 2016 (A, C, E) and 2017 (B, D, F)

A-B) the risk of transmission (i.e. the proportion of replicate ramets of each genotype in which the elicitation of a tissue loss lesion was observed following the application of an inoculant fragment); C-D) the average rate of progression of tissue loss for genotypes with at least three responding replicates; and E-F) the average time until a lesion was observed for genotypes with at least three responding replicates. Genotype designations (given along the $\mathrm{x}$-axis) are identified in Suppl Table 1. The number of replicates for each genotype is given inside or above each bar while error bars indicate +1SE. In A and B, dashed lines show the pooled population risk of infection for each species, asterisks indicate genotypes that differed significantly from this expected transmission risk, and crosses indicate zero transmission observed for that replicate (though power for the Pearson Chi-Squared test was inadequate to conclude that zero differed from expected). 

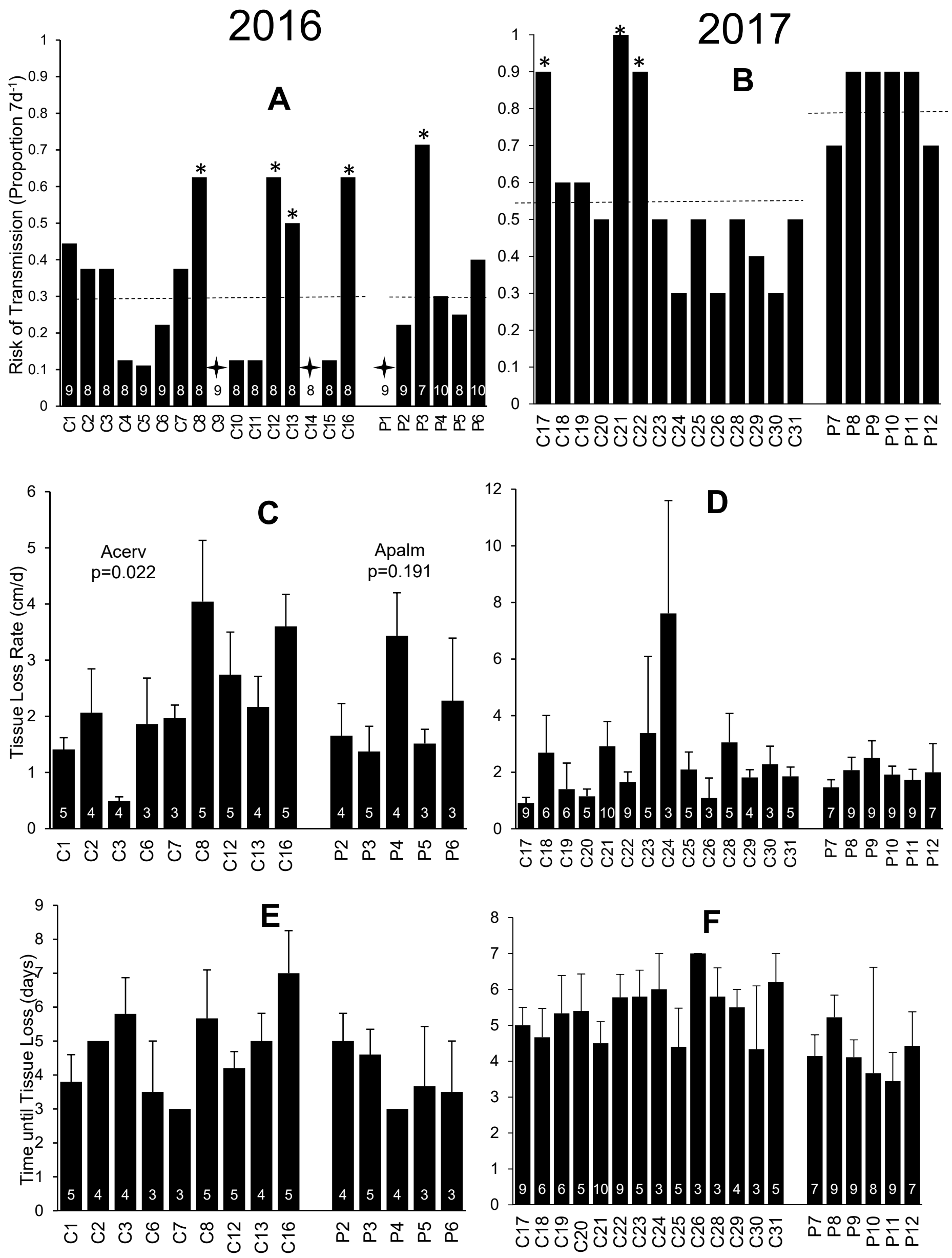